\title{
Using privately owned animals in a study of human subjects
}

Julie Schnepps, a private-practice veterinarian in California, was interested in the human-animal bond, and an opportunity was emerging for her to further that interest. One of Schnepps's clients, Dr. Lucas Roman, was a reconstructive surgeon on a sabbatical leave from Great Eastern University. He suggested to her that it might be of importance to determine if pet-assisted therapy could lower the level of anxiety that many of his teenage patients experienced after undergoing surgery for traumatic facial injuries. Schnepps jumped at the chance but didn't know how or where to begin, so she recruited the help of Dr. Maria Torres, her friend and a laboratory animal veterinarian at Riverbank University, a nearby research university. Schnepps and Torres decided that measuring blood cortisol levels in adolescent patients that did or did not have pet dogs would provide the initial data they needed. As a favor to Torres, Riverbank's clinical laboratory agreed to carry out the cortisol analyses at a minimal cost, which Schnepps would pay using funds from her private practice. Torres did not think that IACUC approval was necessary for the study.

Roman was a faculty member at the Great Eastern University medical school, and his surgical practice was at the Great Eastern Hospital. The hospital and the university were legally separate entities, although medical school students used the hospital for part of their training. Roman received approval for the study from the hospital's Human Research Subjects Committee (analogous to an IACUC) and its Infection Control Committee. Once his patients were postoperatively stable, healing well, yet still hospitalized, they would be brought to a special visiting room where they would be allowed a short, controlled interaction with their pet dog. Blood samples would be collected from the patients before and after the dog visits, and then, after their discharge from the hospital, a blood sample would be collected every time they returned for a postsurgical visit.

The primary interest of Schnepps and Roman was patient stress, as reflected in cortisol levels. They hoped to demonstrate that the teenage patients who had dogs had significantly lower cortisol levels than those without dogs. Given the many variables present, this was meant to be a pilot study that might (or might not) provide a justification for moving forward with more involved research on pet-assisted therapy.

Because the dogs are such a critical part of the study, is IACUC approval needed? If so, which IACUC should review the protocol: Riverbank University's or Great Eastern University's? Roman is a faculty member at Great Eastern University's medical school; as part of their training, medical school students might observe the planned patientanimal interaction on video monitors. Does this fact affect the need for IACUC approval?

\section{RESPONSE}

\section{Patient-pet interactions}

\section{Barbara C. Hansen, PhD}

The described scenario has several aspects. The first is the desire of a private veterinarian to measure cortisol levels in blood samples from pet owners and from people who don't own pets. Though wellintentioned, this notion does not constitute a valid research study of stress levels in adolescent patients who have or do not have pets. There is no need for any IACUC review of this aspect.

The second aspect is the sampling of blood in adolescents before and after a short visit by their own pets in a special room in the hospital and during their postoperative recovery period. Approval of this study by the university's human use committee implies that all concerns related to the inclusion of humans in the study, such as infection risk, dander risk and room sanitation, were addressed to the committee's satisfaction. In my opinion, this aspect of the scenario also does not constitute a well-designed research study of human stress (or even a well-designed pilot study), but if it satisfied the human use committee, then so be it. There is no need for IACUC review of this aspect.

The third aspect is the recording of patient interactions with their own pets (assumedly with the patients' knowledge and consent) and the possibility that medical students might view the video. In my opinion, this does not require IACUC approval for multiple reasons. The patients and their parents or legal guardians have presumably agreed to participate in the study with full knowledge of the patientpet meeting conditions (including the video). There is no animal welfare issue of any sort involved in the scenario provided. There is no research done on the animals, nor will any conclusions regarding the animals be derived. Given the information provided, there is no abuse or risk to the pets themselves beyond normal humanpet interactions. The study is on patients' responses to their own pets.

I see no reason for any IACUC involvement here. If there were an IACUC issue here, however, the only IACUC implicated is the one at Great Eastern University.

Hansen is Professor of Internal Medicine and Pediatrics at Morsani College of Medicine, University of South Florida, Tampa, FL. 